\title{
从线性到非线性的四种基本电子元件 特性普遍公式*
}

\author{
陈栄年王建成
}

(华侨大学物理系, 泉州 362011)

\section{关链调四种基本电子元件、特性普遍公式、线性和非线性}

四种基本电子元件电阻、电容、自感和互感介质从线性各向同性到线性各向异性再到非线 性这三个层次共计 12 个特性普遍公式, 以前只知道其中电阻、自感和互感当介质为线性各向 同性的如下 3 个特性普遍公式(下简称公式)

$$
R^{(0)}=\int \rho^{(0)} \frac{\mathrm{d} l}{S} ; L_{t^{\prime}}^{(0)}-\frac{\mu^{(0)}}{4 \pi} \iint \frac{\mathrm{d} l_{t} \cdot \mathrm{d} l_{t^{\prime}}^{\prime}}{R_{t^{\prime} t}} ; M_{i \ell}^{(0)}-\frac{\mu^{(0)}}{4 \pi} \iint \frac{\mathrm{d} l_{t} \cdot \mathrm{d} l_{\ell}}{R_{\ell \ell}} .
$$

直到 1987 年后, 从电网络场论工作中 ${ }^{[1]}$ 才陆续推导出其余新的 7 个精确公式和 2 个近似公 式. 本文第一次系统地通报上述工作.

\section{1 线性各向异性和非线性的电阻特性普遍公式}

$\hat{\boldsymbol{E}}$ 和 $\hat{\boldsymbol{J}}$ 在线性各向异性电介质中的关系为

$$
\hat{E}_{n}\left(u_{1} u_{2} u_{3} t\right)=\sum_{m=1}^{3} \rho_{m}^{(0)}\left(u_{1} u_{2} u_{3}\right) \hat{j}_{m}\left(u_{1} u_{2} u_{3} t\right)(n-1,2,3) \text {, }
$$

式中 $u_{1}, u_{2}$ 和 $u_{3}$ 为广义正交坐标变量. 当二阶电阻率张量 $\rho_{n m}^{(0)}$ 为对称张量时, 上式中系数 矩阵 $\left[\rho_{n}^{(0)}\right]$ 可化成对角形式的矩阵.

如 $\boldsymbol{j}$ 的流线 $l$ 在三个广义正交坐标面上的投影为 $l_{1}, l_{2}$ 和 $l_{3}$, 则对(1)式作以下积分

$$
\int_{0}^{l} E \cdot \mathrm{d} \boldsymbol{l}-\sum_{n=1}^{3} \sum_{m=1}^{3} \int_{0}^{l_{n}} \rho_{m=}^{(0)} \hat{j}_{m} \mathrm{~d} l_{n},
$$

取 $d \boldsymbol{l}$ 方向平行于该处的 $\boldsymbol{j}$,则

$$
\int_{0}^{l} \hat{E} \cdot \mathrm{d} \boldsymbol{l}-\sum_{n=1}^{3} \sum_{m=1}^{3} \int_{0}^{l_{n}} \rho_{n m}^{(0)} \cos \left(j, e_{\mathrm{n}}\right) \cos \left(\boldsymbol{j}, \boldsymbol{e}_{m}\right) \hat{j} \mathrm{~d} l,
$$

$\boldsymbol{e}_{n}$ 是广义正交坐标线的单位矢量. 上式可表为

$$
\hat{v}-R^{(0)} \hat{p} \text {, }
$$

由式中得出各向异性电阻及其张量的公式各为

$$
R^{(0)}-\int \rho^{(0)} \frac{\mathrm{d} l}{\mathrm{~S}}-\sum_{n=1}^{3} \sum_{m=1}^{3} R_{m}^{(0)} \cos ^{2}\left(j, e_{m}\right),
$$

*䅦建省国然科学基金资助项目. 


$$
R_{m}^{(0)}=\int_{0}^{l_{*}} \rho_{m m}^{(0)} \frac{h_{m} \mathrm{~d} u_{n}}{S_{m}\left(u_{1} u_{2} u_{3}\right)} \quad(n, m=1,2,3),
$$

式中 $h_{n}(n-1,2,3)$ 是广义正交坐标的量度系数, $S_{m}$ 是电阻横截面 $S$ 在 $u_{m}$ 为常数的坐标 面上的投影.

当导电介质为非线性时, (1)式推广为 ${ }^{[2]}$

$$
\hat{E}_{n}-\sum_{m=1}^{3} \rho_{n m}^{(n)} \hat{j}_{m}+\frac{1}{2} \sum_{m=1}^{3} \sum_{p=1}^{3} \rho_{m m p}\left(\hat{i}_{m} \hat{i}_{p}+\hat{i}_{m} \hat{i}_{p}^{*}\right)+\cdots-\sum_{m=1}^{3} \rho_{n m} \hat{i}_{m},
$$

式中电阻率张量 $\rho_{n m}$ 及其非线性第 $k$ 级的 $\rho_{m n}^{(k)}$ 各为

$$
\begin{aligned}
& \rho_{m n}=\rho_{m_{n}}^{(n)}+\rho_{m_{n}}^{(1)}+\cdots+\rho_{m_{n}}^{(k)}+\cdots,
\end{aligned}
$$

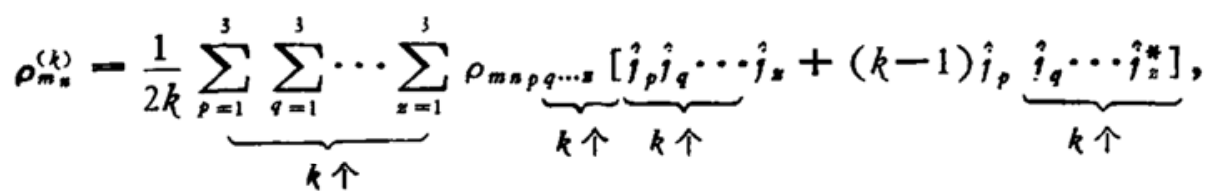

用(4)式替代(1)式,重复推导(2)和(3)式的步骤, 便得非线性电阻及其张量的公式各为

$$
\begin{gathered}
R=\sum_{n=1}^{3} \sum_{m=1}^{3} R_{n m} \cos ^{2}\left(j, e_{m}\right), \\
R_{n m}-\int_{0}^{I_{n}}\left(\rho_{n m}^{(0)}+\rho_{n m}^{(1)}+\cdots+\rho_{m}^{(k)}+\cdots\right) \frac{h_{n} d u_{n}}{S_{m}\left(u_{1} u_{2} u_{3}\right)} \quad(n, m-1,2,3),
\end{gathered}
$$

当 $\left[\rho_{n, i}^{(0)}\right]$ 和 $\left[\rho_{m m}\right]$ 为对称矩阵时, (3)和(8)式各简化为

$$
\begin{gathered}
R_{n n}^{(0)}-\int_{0}^{l_{n}} \rho_{n n}^{(0)} \frac{h_{n} \mathrm{~d} u_{n}}{S_{n}\left(u_{1} u_{2} u_{3}\right)}(n-1,2,3), \\
R_{n n}-\int_{0}^{l_{n}}\left(\rho_{n n}^{(0)}+\rho_{n n}^{(1)}+\cdots+\rho_{n}^{(n)}+\cdots\right) \frac{h_{n} \mathrm{~d} u_{n}}{S_{n}\left(u_{1} u_{2} u_{3}\right)} \quad(n-1,2,3) .
\end{gathered}
$$

\section{2 从线性到非线性的电容特性普遍公式}

当电场 $\hat{E}-E_{0} e^{i \omega r}$ 谐变时,利用下面 3 式:

$$
\begin{gathered}
D=\varepsilon^{(0)} \hat{E}, \\
\hat{j}^{D}=\frac{\mathrm{d} \dot{D}}{\mathrm{~d} t}-i \omega \varepsilon^{(0)} \hat{\boldsymbol{E}}, \\
\hat{I}^{D}=\hat{j}_{a}^{D} S(l),
\end{gathered}
$$

( $\hat{j}_{a}^{D}$ 是 $P^{D}$ 通过 $S(l)$ 的平均密度) 和高斯定理, 得到电容器内电路的位移电流 $P^{D}$ 的复式欧 姆定律 ${ }^{[3]}$

$$
\hat{V}=\hat{Z}^{(c)} \hat{I}^{D},
$$

式中电容复阻抗 $Z^{(c)}$ 与电容器的介质和形状有关.

$$
\hat{\mathbf{Z}}^{(c)}=\frac{1}{i \omega} \int \frac{\mathrm{d} l}{\varepsilon^{(0)}(l) S(l)},
$$

由此得出线性各向同性的电容公式为

$$
C=\frac{1}{\int_{0}^{l}-\frac{\mathrm{d} l}{\varepsilon^{(0)}(l)} \frac{S(l)}{},}
$$


式中 $S(l)$ 是位于电容器内距离正极板为 $l$ 处的一个等位面. (15)式是电容定义式之外能表 达电容普遍特性的另一个电容基本公式.

当电介质为线性各向异性时,(11)式和(12)式分别推广为

$$
\begin{aligned}
& \hat{D}_{n}\left(u_{1} u_{2} u_{3} t\right)=\sum_{m=1}^{3} \varepsilon_{n}^{(0)}\left(u_{1} u_{2} u_{3}\right) \hat{E}_{m}\left(u_{1} u_{2} u_{3} t\right) \quad(n-1,2,3), \\
& \hat{i}_{*}^{D}\left(u_{1} u_{2} u_{3} t\right)-i \omega \sum_{m=1}^{3} \varepsilon_{n m}^{(0)}\left(u_{1} u_{2} u_{3}\right) \hat{E}_{m}\left(u_{1} u_{2} u_{3} t\right) \quad(n-1,2,3) .
\end{aligned}
$$

若 $\left[\varepsilon_{\mathrm{m}}^{(0)}\right]$ 是非奇异矩阵,则 (17)式有唯一解

$$
\hat{E}_{n}=\sum_{m=1}^{3} \varepsilon_{n m}^{(0)-1} \frac{j_{m}^{D}}{i \omega} \quad(n-1,2,3),
$$

式中 $\varepsilon_{n m}^{(0)-1}$ 是逆矩阵 $\left[\varepsilon_{n m}^{(0)}\right]^{-1}$ 中的元素. 对上式作积分

$$
\int_{0}^{l} \hat{E} \cdot \mathrm{d} l=\sum_{n=1}^{3} \sum_{m=1}^{3} \int_{0}^{l_{n}} \varepsilon_{n m}^{(0)-1} \frac{\hat{i}_{m}^{D}}{i(c o} \mathrm{d} l_{n},
$$

并把下列分量关系

$$
\hat{i}_{m}^{D}-\frac{\hat{I}^{D}}{S} \cos \left(j^{D}, e_{m}\right) ; \mathrm{d} l_{n}-\mathrm{d} l \cos \left(j^{\nu}, e_{n}\right)
$$

代人, 表成介质中位移电流 $\hat{I}^{D}$ 的欧姆定律 ${ }^{[4]}$

$$
\hat{v}=\left(\frac{1}{i \omega C}\right)^{0} \hat{I}^{D},
$$

从而得出各向异性倒电容及其张量的公式各为

$$
\begin{aligned}
& \left(\frac{1}{C}\right)^{(0)}-\sum_{m=1}^{3} \sum_{m=1}^{3}\left(\frac{1}{C}\right)_{m}^{(0)} \cos ^{2}\left(j^{D}, e_{m}\right), \\
& \left(\frac{1}{C}\right)_{n m}^{(0)}-\int_{0}^{l_{n}} \varepsilon_{n m}^{(0)-1} \frac{h_{n} \mathrm{~d} u_{n}}{S_{m}\left(u_{1} u_{2} u_{3}\right)} \quad(n, m-1,2,3) .
\end{aligned}
$$

当电介质为非线性时, (16)和(17)式又推广为

$$
\begin{gathered}
\hat{D}_{n}-\sum_{m=1}^{3} \varepsilon_{n m}^{(0)} \hat{E}_{m}+\frac{1}{2} \sum_{m=1}^{3} \sum_{p=1}^{3} \varepsilon_{m m p}\left(\hat{E}_{m} \hat{E}_{p}+\hat{E}_{m} \hat{E}_{p}^{*}\right)+\cdots(n-1,2,3), \\
\hat{i}_{n}^{\nu}=i \omega \sum_{m=1}^{3} \varepsilon_{n m} \hat{E}_{m}(n-1,2,3) .
\end{gathered}
$$

介电常数张量 $\varepsilon_{\mathrm{sm}}$ 及其非线性的第 $k$ 级 $\varepsilon_{m}^{(k)}$ 各为

$$
\begin{aligned}
& \varepsilon_{n m}-\varepsilon_{n m}^{(0)}+\varepsilon_{n m}^{(1)}+\cdots+\varepsilon_{n m}^{(k)}+\cdots, \\
& \varepsilon_{k m}^{(k)}-\frac{1}{2 k} \underbrace{\sum_{p=1}^{3} \sum_{q=1}^{3} \cdots \sum_{x=1}^{3} \varepsilon_{n m p q \cdots a}}_{k \uparrow} \underbrace{\left[\hat{j}_{p} \hat{i}_{q} \cdots \hat{i}_{x}\right.}_{k \uparrow}+(k-1) \underbrace{\left.\hat{i}_{p} \hat{j}_{q} \cdots \hat{j}_{k}^{*}\right]}_{k \uparrow} \underbrace{\underbrace{}_{k}}_{k \uparrow}
\end{aligned}
$$

用 (23)式替代(17)式, 重复推导(20)和(21)式的步骤 ${ }^{[5-7]}$, 便得非线性的倒电容及其张量的公 武各为

$$
\left(\frac{1}{C}\right)=\sum_{m=1}^{3} \sum_{m=1}^{3}\left(\frac{1}{C}\right)_{n m} \cos ^{2}\left(j^{D}, e_{m}\right)
$$




$$
\left(\frac{1}{C}\right)_{n m}-\int_{0}^{l} \varepsilon_{n m}^{-1} \frac{h_{n} \mathrm{~d} u_{n}}{S_{m}\left(u_{1} u_{2} u_{3}\right)} \quad(n, m-1,2,3) .
$$

当 $\left[\varepsilon_{, m}^{(0)}\right]$ 和 $\left[\varepsilon_{* m}\right]$ 为对称矩阵时, (21)和 (25)式简化为

$$
\begin{gathered}
\left(\frac{1}{C}\right)_{. *}^{(0)}-\int_{0}^{l_{n}} \frac{h_{n} \mathrm{~d} u_{n}}{\varepsilon_{n n}^{(0)} S_{n}\left(u_{1} u_{2} u_{3}\right)} \quad(n-1,2,3), \\
\left(\frac{1}{C}\right)_{n}-\int_{0}^{l_{*}} \frac{h_{n} \frac{\mathrm{d} u_{n}}{\left(\varepsilon_{n n}^{(0)}+\varepsilon_{n n}^{(1)}+\cdots+\varepsilon_{n i}^{(n+}+\cdots\right) S_{n}\left(u_{1} u_{2} u_{3}\right)} \quad(n-1,2,3) .}{}
\end{gathered}
$$

\section{3 线性各向异性和非线性的自感和互感特性普遍公式}

在均匀各向异性介质中磁场基本方程组为

$$
\nabla \times H=j ; B-\nabla \times A ; B_{n}-\sum_{m=1}^{3} \mu_{n m}^{(0)} H_{m}(n-1,2,3),
$$

解得各向异性磁矢势分量 $A_{n}$ 的积分公式为 ${ }^{[8.9]}$

$$
A_{n}(\boldsymbol{x})=\frac{1}{4 \pi \sqrt{\left|\mu_{i k}^{(0)}\right|}} \sum_{m=1}^{3} \Delta_{n m} \int \frac{j_{m}\left(\boldsymbol{x}^{\prime}\right) \mathrm{d} V^{\prime}}{\left(\sum_{i=1}^{3} \sum_{k=1}^{3} \mu_{i k}^{(0)-1} R_{i} R_{k}\right)^{1 / 2}}(n-1,2,3),
$$

式中 $\boldsymbol{R}-\boldsymbol{x}-\boldsymbol{x}^{\prime}$, 而 $\left|\boldsymbol{\mu}_{i k}^{(0)}\right|$ 和 $\boldsymbol{\mu}_{i k}^{(0)-1}$ 分别代表磁导率张量 $\boldsymbol{\mu}_{i k}^{(0)}$ 的行列式和逆矩阵 $\left[\boldsymbol{\mu}_{i k}^{(0)}\right]^{-\boldsymbol{s}}$ 中的元素, $\Delta_{n m}$ 代表行列式 $\left|\mu_{m}^{(0)}\right|$ 中元素为 $\mu_{m}^{(0)}$ 的代数余子式.

正弦稳态闭合回路中,按法拉第定律有

$$
\oint \hat{\boldsymbol{E}} \cdot \mathrm{d} \boldsymbol{l}-i \omega \oint \hat{\boldsymbol{A}}^{(\boldsymbol{L})} \cdot \mathrm{d} \boldsymbol{l}+i \omega \oint \hat{\boldsymbol{A}}^{(\boldsymbol{M})} \cdot \mathrm{d} \boldsymbol{l} \text {. }
$$

若回路 $l$ 由 $N$ 个支路组成并有 $G$ 个外支路与之连接而有磁耦合的作用, 上式便写成

$$
\sum_{g=1}^{N} \int \hat{E}_{g} \cdot \mathrm{d} l_{z}-i \omega \sum_{g=1}^{N} \sum_{n=1}^{3} \int \hat{A}_{l z, n}^{(L)} \mathrm{d} l_{s, n}+i \omega \sum_{e=1}^{G} \sum_{g=1}^{N} \sum_{n=1}^{3} \int \hat{A}_{e g, n}^{(M)} \mathrm{d} l_{z, . .}
$$

把(28)式两边都乘以因子 $e^{i \infty t}$ 并应用于某一支路上, 代人上式右边中, 经整理可表成电路方 程形式 ${ }^{[8,9]}$

$$
\hat{V}_{l}=\hat{Z}_{l l}^{(L)} \hat{I}_{l^{\prime}}+\sum_{e=1}^{G} \hat{Z}_{e l}^{(M)} \hat{I}_{l},
$$

从而得出各向异性自感和互感以及它们张量的公式各为

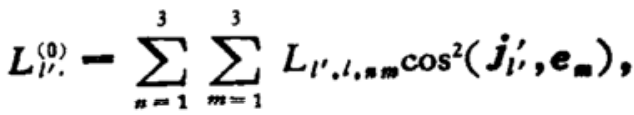

$$
\begin{aligned}
& M_{e l}^{(0)}-\sum_{m=1}^{3} \sum_{m=1}^{3} M_{e l, m_{m}}^{(0)} \cos ^{2}\left(j_{e}, e_{m}\right)
\end{aligned}
$$

和

$$
\begin{aligned}
& L\left(\ell_{l, \ldots-m}-\frac{\Delta_{n m}}{4 \pi \sqrt{\left|\mu_{i k}^{(0)}\right|}} \oint_{l} \oint_{l^{\prime}} \frac{\cos \left(j_{l}, e_{n}\right)}{\cos \left(j_{l^{\prime}}^{\prime}, e_{m}\right)} \frac{\mathrm{d} l_{l} \mathrm{~d} l_{l}^{\prime}}{\left.\left(\sum_{i=1}^{3} \sum_{k=1}^{3} \mu_{i k}^{(0)-1} R_{l^{\prime} l, i} R_{l^{\prime} l_{k}}\right)\right)^{1 / 2}}\right. \\
& \left(n_{1} m-1,2,3\right),
\end{aligned}
$$




$$
\begin{aligned}
M_{e l, m m}^{(0)}= & \frac{\Delta_{n m}}{4 \pi \sqrt{\left|\mu_{i k}^{(0)}\right|}} \oint_{1} \int_{l_{e}} \frac{\cos \left(j_{l}, e_{n}\right)}{\cos \left(j_{c}^{\prime}, e_{m}\right)} \frac{\mathrm{d} l_{l} \mathrm{~d} l_{c}^{\prime}}{\left(\sum_{i=1}^{3} \sum_{k=1}^{3} \mu_{i k}^{(0)-1} R_{e l, i} R_{e l, k}\right)^{1 / 2}} \\
& (n, m-1,2,3) .
\end{aligned}
$$

当磁介质为非线性时, $\hat{B}$ 与 $\hat{H}$ 关系为

$$
\hat{B}_{n}=\sum_{m=1}^{3} \mu_{n m} \hat{H}_{m} \quad(n-1,2,3) \text {. }
$$

现在 $\mu_{n m}$ 已不再是恒量, 而是磁场 $\hat{H}$ 的函数

$$
\mu_{n m}-\mu_{n m}^{(0)}+\frac{1}{2} \sum_{p=1}^{3} \mu_{n m p}\left(\hat{H}_{p}+\hat{H}_{p}^{*}\right)+\cdots,
$$

若在一定条件下,能把 $\mu_{n=.}$ 近似看成一个恒量, 用 $\mu_{n=0}$ 表示. 亦即能把上式右边从第二项 起以后所有各项用一个近似恒量 $\Delta$ 来表示, 则 $\mu_{m n_{0}}-\mu_{* m}^{(0)}+\Delta$. 于是, 在(32)和(33)式中把 $\mu_{i k}^{(0)}$ 替换 $\mu_{i k 。}$ 后, 便是非线性的自感和互感的近似工作.

\section{4 结 束 语}

上述非线性的自感和互感的近似公式, 实际上只是保留这个问题, 让有兴趣者继续研究. 此外, 四种基本电子元件介质为三层次的全部特性普遍公式已经全部齐全. 这不仅在理论上 完成了对四种基本电子元件整体和全面的特性认识, 而且在实用上由于覆盖了由线性到非线 性的所有介质,可适于各种需要的应用,对推动电子元件科学的发展有其深远意义.

\section{参考文献}

[1]陈秝年、何煜光、陈洁, 网络现代场论, 电子工业出版社, 北京, 1991,235-241;152-156;170-176;248-254;270284.

[2]陈架年、总建图,电子学报, 1989, 17(1): 125 - 127 .

[ 3] 陈采年、何煜光,华侨大学学报, $1989,10(4): 372-381$.

[4] 陈采年,电子科学学刊, $1987,9(1): 85-89$.

15]陈省年,电子学报, $1987,15(4): 116-119$.

[6] 陈桑年,科学通报, $1991,36(1): 24-27$.

[7] Chen Xin-nisn (陈桑年), Chinese Science Bullerin, 1992, 37 (6): 464-468.

183 陈藥年、陈洁, 电子科学学刊, $1991,13(2)$ : 159-168.

[9] Chen Xin-nian (陈采年), Chen Jie (陈 洁), Journal of Electronics (China), 1991, 8 (3): $247-257$. 